\title{
Statistical Shape Modeling Using MDL Incorporating Shape, Appearance, and Expert Knowledge
}

\author{
Aaron D. Ward and Ghassan Hamarneh \\ Medical Image Analysis Lab, \\ School of Computing Science, Simon Fraser University, Canada \\ \{award, hamarneh\}@cs.sfu.ca \\ http://mial.cs.sfu.cal
}

\begin{abstract}
We propose a highly automated approach to the point correspondence problem for anatomical shapes in medical images. Manual landmarking is performed on a small subset of the shapes in the study, and a machine learning approach is used to elucidate the characteristic shape and appearance features at each landmark. A classifier trained using these features defines a cost function that drives key landmarks to anatomically meaningful locations after MDL-based correspondence establishment. Results are shown for artificial examples as well as real data.
\end{abstract}

\section{Introduction}

In the study of the relationship between anatomical shapes and pathological conditions, it is useful to explore and quantify anatomical shape variability. To this end, the point correspondence problem must be solved: a mapping must be established between points that represents an anatomically meaningful correspondence, to ensure meaningful shape statistics. The Minimum Description Length (MDL) approach to this problem has received considerable attention in the research community 1. Briefly, this approach is a means of evaluating a chosen point correspondence by measuring the information theoretic cost of transmitting the shape model resulting from the correspondence. A previous study revealed that the MDL approach exceeds other current approaches in its agreement with landmarks placed by a human expert [2]. However, for correspondences established between human brain ventricles, this study found that the mean disagreement between landmarks placed by MDL and expert landmarks was $\approx 4 \mathrm{~mm}$; a significant error. Row 1 of figure 2 illustrates this problem; although the established correspondence appears good according to the coloured visualization, highlighting specific point correspondences reveals errors at the peak apexes, particularly at the small peak. Since this peak represents little information content, MDL's cost function tends to de-emphasize it.

The fundamental issue here is that saliency is not necessarily encoded by an information theoretic compactness measure. There exists research into integrating geometry into the MDL process 34 . The idea behind these approaches is to 
represent the shapes in terms of some geometric measurement such as curvature, and run MDL on this representation. These approaches are steps in the right direction and speak to the need for the integration of geometric information with MDL. However, they suffer the shortcomings of the arbitrary choice of geometric features and their uniform use throughout the object surface, failing to consider that different surface points may be characterized by different features.

We propose the use of expert knowledge, in the form of a small set of landmarked training examples, to guide the selection of shape and appearance features. These features are used in guiding MDL-based point correspondence to solutions that correspond with human intuitions of point saliency. Since we expect that human anatomists establish meaningful point correspondence based both on local shape and appearance information (from the underlying medical images), we acquire shape and appearance information about the points chosen by the user during the training phase. We automatically learn, for each landmark, the specific shape and appearance features that best distinguish the landmark. We then use the learned features to guide the process of correspondence establishment toward a solution that agrees with human intuition. There exists an abundance of expert knowledge about anatomically meaningful landmarks, and our philosophy is that this information should not be ignored in a landmarking approach.

\section{Methods}

\subsection{Method Overview}

At a high level, the overall process used in this work consists of two steps, given by the boxes in figure 1] (1) acquiring knowledge about distinguishing shape and appearance features for each expert-chosen landmark ("Training"), and (2) applying that knowledge to drive the point correspondence process toward a better solution ("Correspondence via MDL + landmark features").

The goal of the training step is to find, for each landmark chosen by the user, a set of shape and appearance features that, when utilized in the point correspondence process, (1) drives landmarks toward anatomically meaningful targets as indicated by the user, and (2) reduces as much as possible the chances of a landmark being driven toward a wrong target having similar shape and appearance features to those indicated by the user. The high-level process used in training is to select a subset of shape and appearance features (in a subset, some features are used and some not) that best satisfies the above criteria. To this end, we have designed a cost function that evaluates each subset. The cost function is a combination of two types of errors: a target error $\epsilon_{t}$ and a basin width error $\epsilon_{b} . \epsilon_{t}$, for a given subset of features, is inversely related to the accuracy with which a classifier selects target contour points in the training set as belonging to the class of expert-labelled landmarks. Ideally, the classifier would select only the expert-selected points as targets, yielding $\epsilon_{t}=0 . \epsilon_{b}$ measures, for a given subset of features, the spacing between detected targets along the contour. Ideally, this spacing should be as large as possible in order to eliminate confusion between 


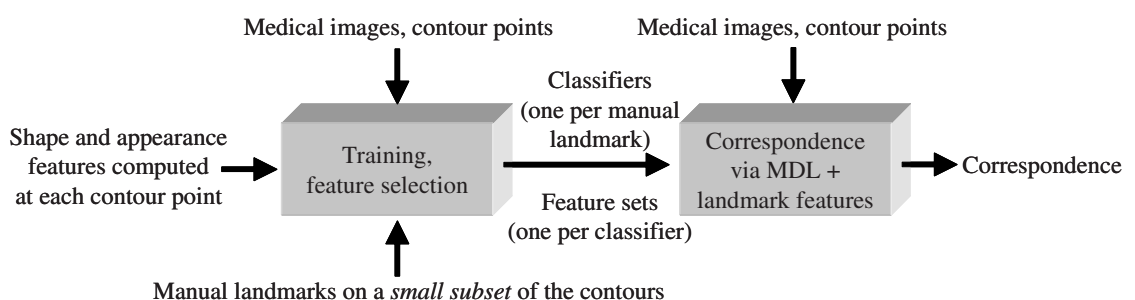

Fig. 1. The overall process followed in this work. See text for details.

targets during correspondence establishment, when landmarks are driven toward the nearest targets.

The goal of the correspondence establishment step is to find a point correspondence resulting in a shape model that is as compact as possible while still anatomically meaningful. To this end, we iteratively minimize the MDL cost function until convergence, which provides a good initialization for a subsequent minimization of a new cost function derived from the classifiers and feature subsets learned during training. This new cost function drives key landmarks to anatomically meaningful locations, according to information gained in training.

\subsection{Training}

Here, we explain the training step in more detail. Given a set of medical images and contour points lying on the boundaries of segmentations of an object of interest on each medical image, we manually collect $n$ landmarks $l_{i j}, i \in$ $\{1, \ldots, n\}, j \in\{1, \ldots, m\}$ on each of a small number $m$ training examples. To this end, we present the user with not only the object contours, but also with the underlying medical images. The rationale for this is that the user may select points based on both shape and appearance features of the object.

Next, for each landmark, we train a classifier, using the best possible subset of shape and appearance features to distinguish the landmark as best as possible from all of the other points on the training objects' contours. The result is $n$ different classifiers, one for each landmark. For the moment, we treat the feature selection problem [5] of choosing the best possible subset of features as a black box; we will return to it in section 2.4.

\subsection{Correspondence Establishment}

Given a set of classifiers (one per landmark) and feature subsets (one per classifier), each classifier assigns each contour point on all contours to one of two classes: points similar to the landmark chosen by the user (which we shall denote class $U)$, and all of the other contour points (class $\tilde{U})$. For illustrative purposes here, we represent curve points parameterized from beginning to end as strings of digits. For example, curve 0000000000000000 has 16 points, each labelled with a zero. We denote points belonging to $U$ with ones and points belonging to $\tilde{U}$ with 
zeros. Consider, for illustrative purposes, a case where a classifier, for one landmark, given a curve and feature subset outputs 0000001110000000 (indicating that three points on the curve are similar to the user's chosen landmark). We need a function such that, given a position $x$ on the curve, the cost function returns the distance to the point best matching the features of the user's chosen landmark. To do this, we first thin the cluster of ones, yielding 0000000100000000 . We then take the distance transform of the result, yielding 7654321012345678. We denote the result of this distance transform, for landmark $i$ on curve $j$ as $c_{i j}(x)$. Thus $c_{i j}(x)$ is a function for landmark $i$ on a shape $j$ that drives landmarks to points similar to user landmarks. Consider a case where MDL deposits a landmark at a location $x_{0}, 3$ positions away from the correct target on the curve; $c_{i j}\left(x_{0}\right)=3$. An energy minimization procedure using a cost function based on $c_{i j}(x)$ will thus drive points to the bottoms of the basins given by the cost functions $c_{i j}(x)$. More specifically, once MDL converges, we locate, on the first contour, the points $m_{i 1}$ corresponding to $l_{i 1}, \forall i=1 \ldots n$, as well as their corresponding points $m_{i j}$ in all of the other contours. We then continue energy minimization using the following cost function based on $c_{i j}(x): \epsilon_{e}=\sum_{i=1}^{n} \sum_{j=1}^{m} c_{i j}\left(m_{i j}\right)$. Using the description length as the cost function in an energy minimization scheme intended to establish point correspondence results in a remarkably good, but imperfect, point correspondence. This good point correspondence serves as an excellent initialization for a gradient descent optimization of $\epsilon_{e}$.

\subsection{Feature Selection}

Here, we explain our approach to feature selection during the training step. We use a cost function to evaluate each subset, and choose the subset with the lowest cost. The cost function is a combination of a target error $\epsilon_{t}(p, i)$ and a basin width error $\epsilon_{b}(p, i)$, for a subset $p$ and a given landmark $i$.

The classifier is trained using all of the user's selected points in class $U$, and all other points in class $\tilde{U}$. For example, consider 5 training shapes, each with 100 contour points. Consider a feature subset involving curvature and its first derivative. We thus have 500 points in 2D feature space, 5 of which (chosen by the user) are in $U$, the remainder in $\tilde{U}$. A classifier is trained on this data, attempting to find a decision boundary that best separates the two classes. All points are then reclassified into classes $U$ and $\tilde{U}$ according to this classifier (in the ideal case, the point classifications do not change, but this is unlikely with real data).

The subset is then assessed according to the output of the classifier. The target error is defined as $\epsilon_{t}(p, i)=\sum_{j=1}^{m} d\left(l_{i j}, \hat{u}_{i j}\right)$, where $d(p, q)$ is the geodesic distance along the object contour between points $p$ and $q$, and $\hat{u}_{i j}$ is the geodesically nearest point in class $U$ on the contour to $l_{i j}$. For example, if the user's selected landmark $i$ (indicated with a 1) is 0000000100000000 and a classifier trained using subset $p$ yields a classification of 0000000000100000 on shape $j$ (the 1 is 3 positions away from the user's landmark), then $d\left(l_{i j}, \hat{u}_{i j}\right)=3$. If the classification were identical to the user's selection, 0000000100000000, then $d\left(l_{i j}, \hat{u}_{i j}\right)=0$. This measure indicates how far the classifier, given a shape 
subset, will move a landmark off target. The basin width error is defined as $\epsilon_{b}(p, i)=\sum_{j=1}^{m}\left(\frac{N_{p}}{2}-d\left(\hat{u}_{i j}, u_{i j}\right)\right)$, where $N_{p}$ is the number of points on the longest contour in the training set (and thus the maximum basin width error is $\frac{N_{p}}{2}$ ) and $u_{i j}$ is the geodesically nearest point in class $U$ on the contour to $\hat{u}_{i j}$. For example, if the user's selected landmark $i$ is 0000000100000000 and a classifier trained using subset $p$ yields a classification of 010000010000100 on shape $j, d\left(\hat{u}_{i j}, u_{i j}\right)=5$. A classification of 0001000101000000 yields $d\left(\hat{u}_{i j}, u_{i j}\right)=2$. Clearly the former is to be preferred over the latter; the latter results in a possibly confusing minimum value of $\epsilon_{e}$ during correspondence establishment. For each subset $p$ for a landmark $i$, we compute an overall error measure $\epsilon(p, i)$, which combines $\epsilon_{b}(p, i)$ and $\epsilon_{t}(p, i)$ :

$$
\underset{p}{\operatorname{argmin}} \epsilon(p, i)= \begin{cases}\epsilon_{t}(p, i)+\frac{\epsilon_{b}(p, i)}{\frac{N_{p}}{2}+1} & \text { if } \exists \epsilon_{t}(p, i)<t \wedge \exists \epsilon_{b}(p, i)<\frac{N_{p}}{2}-t \\ \frac{\epsilon_{t}(p, i)}{\alpha\left(\epsilon_{t}(p, i)-t\right)+1}+\epsilon_{b}(p, i) & \text { otherwise }\end{cases}
$$

In equation 1] $t$ is a threshold specifying desired quantities for $\epsilon_{t}(p, i)$ and $\epsilon_{b}(p, i)$. The basic idea is that if $\epsilon_{t}(p, i)$ and $\epsilon_{b}(p, i)$ are sufficiently small, it is desirable for $\epsilon_{t}(p, i)$ to dominate the cost function; it is a hard constraint that we choose the subset with the smallest value of $\epsilon_{t}(p, i)$ in this case. If this condition is not met, then the cost function becomes a nonlinear combination of $\epsilon_{t}(p, i)$ and $\epsilon_{b}(p, i)$ such that the dominance of $\epsilon_{t}(p, i)$ diminishes as $\epsilon_{t}(p, i)$ grows. This avoids the choice of subsets with relatively large $\epsilon_{t}(p, i)$ and small $\epsilon_{b}(p, i)$ in cases where the smallest possible $\epsilon_{t}(p, i) \geq t$. The rate at which the dominance of $\epsilon_{t}(p, i)$ diminishes is directly proportional to $\alpha$, which was set to $10^{-2}$ for all experiments in this paper. The threshold $t$ can be set automatically by iterating MDL until convergence and then computing the maximum geodesic distance between any landmark and its correct position given by the training data.

Shape features used in our experiments include curvature, the absolute value of the first derivative of curvature, and the local area integral invariant [6]. Appearance features include the average image intensity in a circular region centered at each contour point, the average intensity along a segment extending normal to the contour into the object, and the average intensity along a segment extending normal to the contour out of the object. Each feature was computed at a variety of scales, and equation 1 was minimized by brute force. Note that our method does not dictate the specific choices of these features; the features used in this paper are chosen to exemplify the effectiveness of the method.

\section{Results}

Figure 2 qualitatively compares our method to the performance of standard MDL on rectangles (bottoms cut off for space considerations) with two protruding peaks, one small and one large. Row 1 of figure 2 shows MDL's performance, and row 2 shows the performance of our algorithm trained using a single example with two training points, indicated by the gray diamonds. For the small 


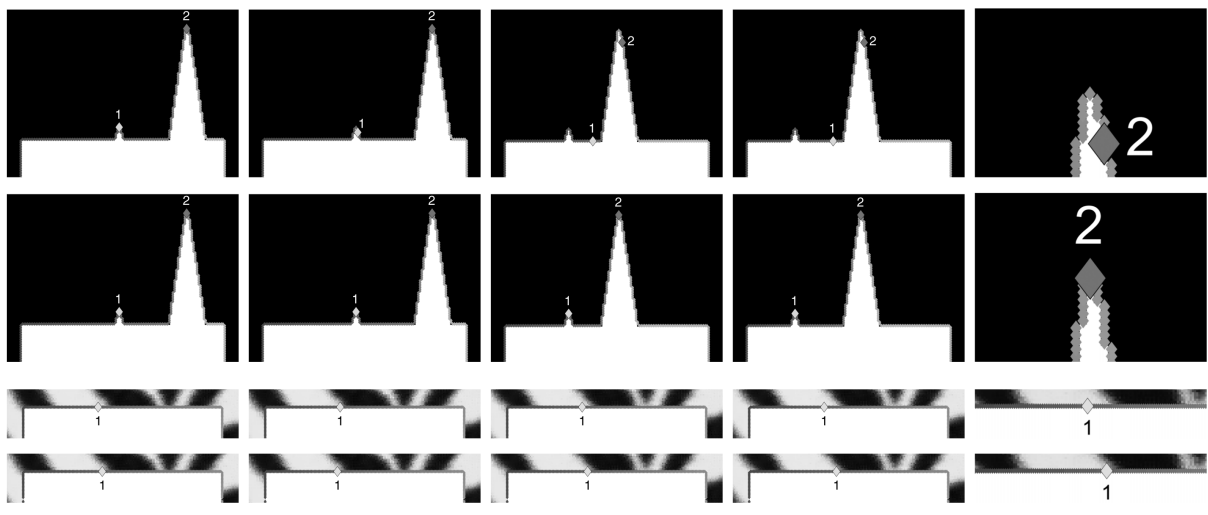

Fig. 2. Comparison of correspondences established by MDL (rows 1 and 3) with those established by our method (rows 2 and 4 ) on artificial examples. Corresponding points have the same colour (see electronic version). The points of interest are indicated by diamonds in different shades of gray, with numbers indicating correspondence.

peak, minimization of equation 1 resulted in the selection of curvature and the absolute value of its derivative as features, as expected. Interestingly, for the large peak, curvature features were not selected; the local area integral invariant was chosen instead, because this feature differentiates the large peak from the small peak, thus yielding a lower $\epsilon_{b}(p, i)$ error. Figure 2 also shows the performance of MDL versus our method for establishing correspondence on a rectangle (bottoms cut off for space considerations), with a point of interest defined by texture. Not surprisingly, standard MDL fails to do this (row 3) since it does not use appearance information (mean correspondence error 4). Our method (row 4) established perfect correspondence using the average local intensity value.

Figure 3 qualitatively compares standard MDL to our method on the corpus callosum (CC; a brain structure), as segmented from MR images. Row 1 shows the result from standard MDL, with diamonds indicating corresponding points of interest. These points should lie on the tip of the rostrum (the apex of the "hook" of the CC on the anterior side; see dark gray diamond in row 2, column 1 ), and at the interface between the $\mathrm{CC}$ and the fornix, which emanates below the $\mathrm{CC}$ in the middle of its body (see light gray diamond in row 2 , column 1 ). In training, the system chose curvature and the absolute value of its derivative as features characterizing the rostrum, and the average intensities along segments normal to the contour emanating into and out of the object to characterize the fornix. Training was performed on 5 examples, with testing on 15 (4 examples shown due to space constraints). Figure 3 also compares standard MDL to our method for a point of interest on the right brain ventricle as segmented from MR images. The third row shows standard MDL, and the fourth row shows our method. Training was performed on 5 examples, with testing on 14 (4 examples shown). Features selected for this point were curvature and its derivative, and the local area integral invariant. 

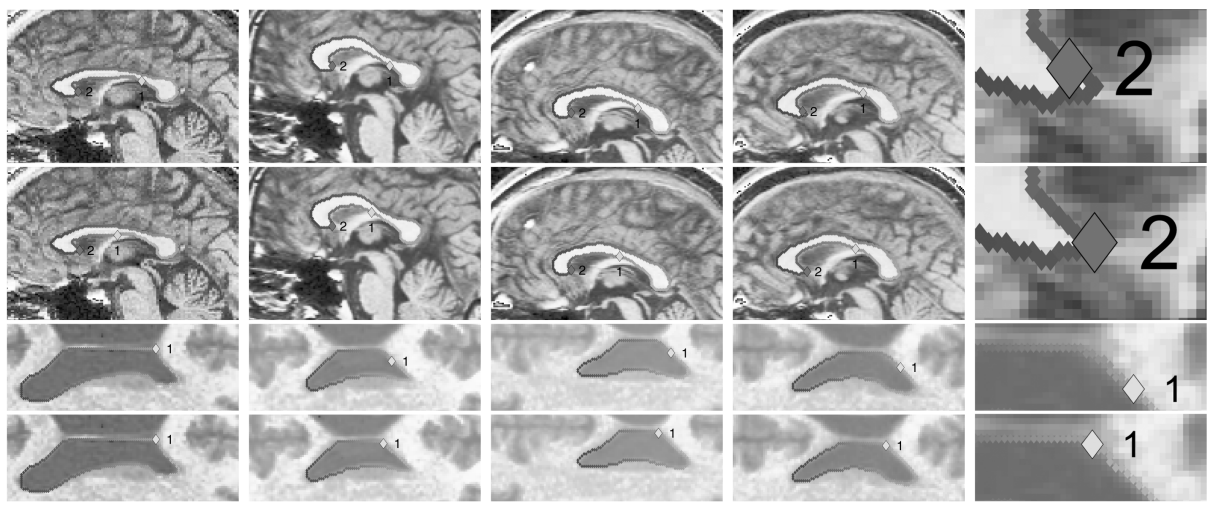

Fig. 3. Comparison of correspondences established by MDL (rows 1 and 3) with those established by our method (rows 2 and 4) on anatomically meaningful points on the corpus callosum and ventricles. Corresponding points have the same colour (see electronic version). The points of interest are indicated by diamonds in different shades of gray, with numbers indicating correspondence.

Table 1 shows training times, correspondence times, and mean geodesic landmark errors (defined as the mean of the geodesic distances between landmarks and their correct, manually-landmarked locations). Note that the correspondence times shown for our method include the time required to perform the MDL-based optimization. Our method shows a decrease in landmarking error, and training and correspondence execute reasonably quickly (on a $2.4 \mathrm{Ghz} \mathrm{AMD}$ Opteron CPU). Note that for the ventricles, the mean error for our method is skewed by outliers; the median error for standard MDL is 6.0 pixels, compared to a median error of 1.5 pixels for our method.

Table 1. Training and correspondence times, and errors, shown as (standard MDL, our method). $\mathrm{LP}=$ large peak, $\mathrm{SP}=$ small peak, $\mathrm{R}=$ rostrum, $\mathrm{F}=$ fornix.

\begin{tabular}{|l|l|l|l|}
\hline \hline & Training (mm:ss) & Correspondence (mm:ss) & Error (pixels) \\
\hline Two peaks & (NA, 01:12) & $(00: 27,01: 17)$ & $(3.40,0.00), \mathrm{LP}$ \\
& & & $(10.0,0.00), \mathrm{SP}$ \\
Texture & $(\mathrm{NA}, 00: 48)$ & $(00: 27,00: 57)$ & $(4.00,0.00)$ \\
CC & $(\mathrm{NA}, 03: 42)$ & $(02: 07,11: 30)$ & $(1.20,0.65), \mathrm{R}$ \\
& & & $(4.50,1.90), \mathrm{F}$ \\
Ventricle & $(\mathrm{NA}, 01: 20)$ & $(00: 55,02: 10)$ & $(5.30,3.20)$ \\
\hline \hline
\end{tabular}

\section{Conclusions}

We have demonstrated the efficacy of a hybrid system for point correspondence establishment, using cost functions measuring model compactness (MDL) and deviation from anatomically meaningful landmarks indicated by the user. 
Although there exist previous approaches incorporating machine learning [7, to the best of our knowledge, ours is the first incorporation of machine learning to elicit local, characteristic features of anatomically meaningful points, and to make use of those features in a MDL-based approach to the correspondence problem. We demonstrate our approach on artificial and real data, showing that it runs reasonably quickly, and automatically reports the features that best characterize each chosen point. A general conclusion that can be drawn from this work is that description length alone is an insufficient criterion for establishing anatomically meaningful correspondences between shapes. It is evident from our results that a correspondence resulting from a shape model with larger than minimum description length yields a more meaningful correspondence. We hypothesize that the explanation for this observation is that the MDL criterion fails to capture all types of features which are salient to humans. Features which are insignificant in terms of their effect on the cost of transmitting the shape model are more likely to be ignored by a purely MDL-based shape correspondence method. Future work involves augmenting this system with more features, testing different types of classifiers, and using a more intelligent approach to minimize equation 1 to handle a larger number of features, including saliency features [8], for example. This approach also requires validation on large data sets with ground truth expert correspondences. We are also exploring the extension of this work to $3 \mathrm{D}$, which involves $2 \mathrm{D}$ definitions of the geodesic distances used to compute $\epsilon_{t}$ and $\epsilon_{b}$, and trivial 2D definitions of the thinning and distance transform operations (on surfaces) used to define $\epsilon_{e}$. Also, the integration of the optimization of the MDL cost function and $\epsilon_{e}$ is being explored with the aim of producing a fully hybrid approach. It is also of interest to explore the utility of this approach in elucidating parts of the decision process used by experts to locate landmarks on medical images, by discerning characterizing shape and appearance features not obvious to the layperson or medical trainee.

\section{References}

1. Davies, R., et al.: A minimum description length approach to statistical shape modeling. IEEE TMI 21(5), 525-537 (2002)

2. Styner, M., et al.: Evaluation of 3D correspondence methods for model building. In: Taylor, C.J., Noble, J.A. (eds.) IPMI 2003. LNCS, vol. 2732, pp. 63-75. Springer, Heidelberg (2003)

3. Thodberg, H., et al.: Adding curvature to minimum description length shape models. BMVC 2, 251-260 (2003)

4. Heimann, T., et al.: Implementing the automatic generation of $3 \mathrm{D}$ statistical shape models with ITK. In: Open Science Workshop at MICCAI, Copenhagen (2006)

5. Guyon, et al.: An introduction to variable and feature selection. Journal of Machine Learning Research 3, 1157-1182 (2003)

6. Manay, S., et al.: Integral invariants for shape matching. IEEE PAMI 28(10), 1602$1618(2006)$

7. Pitiot, A., et al.: Learning shape correspondence for n-D curves. IJCV 71(1), 71-88 (2007)

8. Kadir, T., et al.: Scale, saliency and image description. IJCV 45(2), 83-105 (2001) 